\title{
Editorial
}

\section{Special Issue on Intelligent Biomedical Data Analysis and Processing}

\author{
Deepak Gupta ${ }^{\mathrm{a}, *}$, Oscar Castillo ${ }^{\mathrm{b}}$ and Ashish Khanna ${ }^{\mathrm{a}}$ \\ ${ }^{a}$ Maharaja Agrasen Institute of Technology, Delhi, India \\ ${ }^{\mathrm{b}}$ Tijuana Institute of Technology, Tijuana, Mexico
}

Today, humankind lives in the age of Information (and) Technology. Information is the key, the power and the engine that moves the world economy. The world is dependent on market data, medical/epidemiologic sets, Internet browsing records, geological survey data, complex engineering models, and so on. Health Sciences are highly dependent on Information Technology. Health Sciences and Biology are very complex fields and have covered a long distance since ancient times. In the early eighties, Artificial Intelligence in Medicine was a primary research area while developing medical expert systems in specialized medical domains to support diagnostic decision-making. The main problems addressed at this early stage of expert system research were concerned with knowledge acquisition, knowledge representation, reasoning, and explanation. Nowdays, there are many modern hospitals and health care institutions, which are well equipped with monitoring and other advanced data collection devices. The need for knowledge on the domain or the data analysis process becomes essential in biomedical applications, as medical decision making needs to be supported by arguments based on basic medical and pharmacological knowledge. The new tool for analyses of biomedical applications is "Intelligent Data Analysis (IDA)".

Intelligent Data Analysis can be defined as specialized statistical, pattern recognition, machine learning,

${ }^{*}$ Corresponding author: Deepak Gupta, Maharaja Agrasen Institute of Technology, Delhi, India. E-mail: deepakgupta@mait.ac.in. data abstraction, and visualization tools for analysis of data and discovery of mechanisms that created the data. The main idea underlying the concept of Intelligent Data Analysis is extracting knowledge from a vast amount of data with many variables representing very complex and non-linear, real-life problems. Moreover, Intelligent Data Analysis can help to start from the raw data, coping with prediction tasks without knowing the theoretical description of the underlying process, classification tasks of new events based on past ones, or modelling the aforementioned unknown process. Deep learning is one of the fast-growing technologies, which could learn, reason, and understand the available data. Recently, we have observed several significant breakthroughs in research on artificial intelligence, including self-driving cars, computer Go, image recognition, speech recognition, and machine translation. Deep Learning has been tremendously successful at object recognition and detection, localization, scene classification, action recognition, and caption generation. For example, Convolutional Neural Networks (CNNs) have become powerful machine-learning models for various vision-based applications to represent mid-level and high-level abstractions obtained from raw data (e.g., medical images). Deep Neural Networks have also gained considerable commercial interest due to the development of new variants using high-performance GPUs.

This special issue focuses on recent advances, challenges, and future perspectives about intelligent data analysis methods applied in biomedical studies in dif- 
ferent knowledge domains. From around thirty-one submitted articles to this particular section, twelve papers were selected based on corresponding reviews. Each paper was reviewed by at least two reviewers and went through at least two rounds of reviews. The brief contributions of these papers are discussed below.

The first paper in this special issue, authored by $J$. Revathi, J. Anitha, D. Jude Hemanth and entitled "An Intelligent Medical Decision Support System for Diagnosis of Heart Abnormalities in ECG Signals", describes Artificial Neural Network-based methods to detect a Bundle Branch Block (BBB) and Myocardial Infarction (MI) from the multi-lead ECG signal. The clinical characteristics of BBB and MI are extracted using the derivative filter and continuous wavelet transform (CWT). The results reveal that intelligent methods can diagnose $\mathrm{BBB}$ and $\mathrm{MI}$ from ECG signals with high accuracy.

The second paper of the special issue, authored by Poonam Chaudharya, Rashmi Agrawal and entitled "Sensory Motor Imagery EEG Classification Based on Non-Dyadic Wavelets using Dynamic Weighted Majority Ensemble Classification", compares three types of an ensemble classification framework for reducing the inference of non-stationary information and improves the classification decision in BCI system. J48, Naive Bayes, Support Vector Machine, and K-Nearest Neighbor classifiers used as base classifiers to classify extracted features and make a diverse ensemble of classifiers. The publically available datasets: BCI Competition IV dataset IIa and BCI Competition III dataset IVa has been used for experimental evaluation and assessment of the proposed model's performance.

The study in the third paper of the special issue, authored by Abdolkarim Saeedi, Mohammad Karimi Moridani, Alireza Azizi and entitled "An Innovative Method for Cardiovascular Disease Detection Based on Non-linear Geometric Features and Feature Reduction Combination", tackles the lack of reliable models and high training times on a publicly available dataset. The work proposes a novel method based on feature reduction combination, using Genetic Algorithm (GA) and Principal Component Analysis (PCA). The authors present eight dominant features in heart sound classification: mean duration of systole interval, the standard deviation of diastole interval, the absolute amplitude ratio of diastole to $\mathrm{S} 2, \mathrm{~S} 1$ to systole and $\mathrm{S} 1$ to diastole, zero crossings, Centroid to Centroid distance (CCdis) and mean power in the $95-295 \mathrm{~Hz}$ range. According to the experiments done in this study, the developed method can be further explored for real-world heart sound assessments.
The fourth paper of the special issue, authored by Akansha Singh, Ashish Payal and entitled "CAD Diagnosis by Predicting Stenosis in Arteries using the Data Mining Process", proposes a method to diagnose whether the arteries are stenotic or not. Data preprocessing and feature selection has been made on the dataset to improve accuracy. Different supervised classifiers were applied to the selected features. The highest accuracies for left anterior descending (LAD), left circumflex (LCX), and Random Forest obtained the right coronary artery (RCA). Among all the arteries, LAD has the highest accuracy, which means that chances of a person having LAD as stenotic are very high.

The fifth paper of the special issue, authored by J Susymary, P Deepalakshmi and entitled "Machine Learning for Precision Medicine Forecasts and Challenges when incorporating Non-Omics and Omics Data", presents a review of machine learning models for the eventual development of a successful precision medicine forecast model, different strategies for integrating non-omic data and omic data, limitations and challenges in data integration, and future directions for the precision medicine forecasts. The literature also discusses non-omic data, diseases associated with air pollutants, and omic data. It intends to motivate researchers seeking insight beyond their proficiency in the global integrative analytical approach.

The sixth paper of the special issue, authored by Richa Gupta, M.Afshar Alam, Parul Agarwal and entitled "Whale Optimization Algorithm Fused With SVM To Detect Stress In EEG Signals", presents the whale optimization algorithm (WOA), a bio-inspired metaheuristic kernel, integrated with Support Vector Machine (VSM) to detect mental stress from EEG signal. The results obtained using the proposed fused algorithms are compared with available methods. The proposed methodology is economical, comfortable and portable. An accuracy of $91 \%$ is obtained for detecting the stress, considering the data set of 14 subjects.

The seventh paper of the special issue, authored by Ankit Agrawal, Sarsij Tripathi, Manu Vardhan and entitled "Uncertainty Query Sampling Strategies for Active Learning of Named Entity Recognition Task", proposes a new active learning algorithm based on the hybrid query sampling strategy. It considers the sentence similarity along with the final probability value of the model and compared them with four other well-known pool based uncertainty query sampling strategies based active learning approaches for named entity recognition (NER), i.e. least confident sampling, the margin of confidence sampling, the ratio of confidence sampling and 
entropy query sampling strategies. The proposed active learning algorithm performs well and further reduces the annotation cost in comparison to the other sampling strategies based active algorithm in most of the cases.

Feature selection is a crucial stage in the design of a computer-aided classification system for breast cancer diagnosis. The main objective of the eighth paper of the special issue, authored by Shankar Thawkar, Law Kumar Singh, Munish Khanna and entitled "Multiobjective Techniques for Feature Selection and Classification in Digital Mammography", proposes a research design to discover the use of Multi-Objective Particle Swarm Optimization (MOPSO) and Nondominated Sorting Genetic Algorithm-III (NSGA-III) for feature selection in digital mammography. The Pareto-optimal fronts are generated by MOPSO and NSGA-III for two conflicting objective functions, the number of selected features is presented, and mean squared error is used to select optimal features. An artificial neural network (ANN) is used to compute the fitness of the objective functions. The experimental results show that MOPSObased optimization is superior to NSGA-III. MOPSO achieves high accuracy with a 55\% feature reduction. MOPSO based feature selection and classification deliver an efficiency of $97.54 \%$ with $98.22 \%$ sensitivity, 96.82\% specificity, 0.9508 Cohen's kappa coefficient, and area under curve $\mathrm{AZ}=0.983 \pm 0.003$.

The ninth paper of the special issue, authored by Kanksha, Aman Bhaskar, Sagar Pande, Rahul Malik, Aditya Khamparia and entitled "An Intelligent Unsupervised Technique for Fraud Detection in Health Care Systems", presents an analysis of various machine learning techniques to identify Medicare fraud. The isolated forest is selected, which is an unsupervised machine learning algorithm that improves overall performance while detecting fraud based upon outliers. Obtained results were found more promising compared to existing techniques. Around $98.76 \%$ accuracy is obtained using an isolated forest algorithm.

The tenth paper of the special issue, authored by Prerna Sharma, Moolchand Sharma, Divij Gupta, Nimisha Mittal and entitled "Detection of White Blood Cells Using Optimized qGWO”, presents an optimized quantum Grey Wolf Optimization algorithm which is an enhanced version of the Grey Wolf optimization algorithm for feature selection of blood cells. Specifically, the proposed model uses a quantum Grey Wolf optimization algorithm for detection of White Blood cells among the dataset of various type of blood cells. As the standard Grey Wolf Optimization algorithm, this version of the algorithm also works based on the nat- ural hierarchy of wolves. The performance of several classifiers is compared, and the model accuracy approximately reaches $97 \%$. The obtained result shows that the proposed algorithm can find an optimal subset of features and maximize accuracy.

The eleventh paper of the special issue, authored by Hemant P.Kasturiwale, Sujata N Kale and entitled "BioSignal Modelling for prediction of Cardiac Diseases using Intra Group Selection Method", proposes a new machine learning model considering all facet electrocardiogram amplitude (ECG), frequency components, sampling frequency, extraction methods and acquisition techniques. The statistical analysis has been performed on a variety of databases using feature compatibility techniques. The proposed model reaches an average accuracy of 97.87 per cent and is sensitive and precise.

The final (twelfth) paper of the special issue, authored by Akshi Kumar and entitled "Machine Learning for Psychological Disorder Prediction in Indians during COVID-19 Nationwide Lockdown", presents a research study related to the psychological burden among Indians using a COVID-19 Mental Health Questionnaire. After collecting data, it applies predictive analytics and machine learning to identify the likelihood of mental health outcomes using learned features 395 Indian participants. The proposed Psychological Disorder Prediction (PDP) tool uses a multinomial Naïve Bayes classifier to train the model to detect the onset of specific psychological disorder and classify the participants into two predefined categories, namely, anxiety disorder and mood disorder. Experimental evaluation reports a classification accuracy of $92.15 \%$. This automation plays a pivotal role in clinical support as it aims to suggest individuals who may need psychological help.

To conclude, twelve out of a total of thirty-one submitted papers were selected and accepted for inclusion in this special issue. The guest editors hope that the research contributions and findings in this special issue will benefit the readers of the Intelligent Decision technologies Journal, enhance their knowledge and encourage them to work on various aspects of Intelligent Biomedical Data Analysis and Processing.

We want to express our sincere thanks to the Editorsin-Chief, for allowing us to organize this particular issue. The editorial office staff are excellent and we thank them for their support and great work. We are also thankful to all the authors who made this special issue possible and to the reviewers for their thoughtful contributions. 


\section{List of accepted papers}

\begin{tabular}{|c|c|c|}
\hline S. No. & Paper title & Author(s) \\
\hline 1 & $\begin{array}{l}\text { An Intelligent Medical Decision } \\
\text { Support System for Diagnosis of } \\
\text { Heart Abnormalities in ECG } \\
\text { Signals }\end{array}$ & $\begin{array}{l}\text { J. Revathi, J. } \\
\text { Anitha and D. Jude } \\
\text { Hemanth }\end{array}$ \\
\hline 2 & $\begin{array}{l}\text { Sensory Motor Imagery EEG } \\
\text { Classification Based on } \\
\text { Non-Dyadic Wavelets using } \\
\text { Dynamic Weighted Majority } \\
\text { Ensemble Classification }\end{array}$ & $\begin{array}{l}\text { Poonam } \\
\text { Chaudharya, } \\
\text { Rashmi Agrawal }\end{array}$ \\
\hline 3 & $\begin{array}{l}\text { An Innovative Method for } \\
\text { Cardiovascular Disease Detection } \\
\text { Based on Non-linear Geometric } \\
\text { Features and feature reduction } \\
\text { combination }\end{array}$ & $\begin{array}{l}\text { Abdolkarim Saeedi, } \\
\text { Mohammad Karimi } \\
\text { Moridani, Alireza } \\
\text { Azizi }\end{array}$ \\
\hline 4 & $\begin{array}{l}\text { CAD diagnosis by predicting } \\
\text { stenosis in arteries using the data } \\
\text { mining process }\end{array}$ & $\begin{array}{l}\text { Akansha Singh and } \\
\text { Ashish Payal }\end{array}$ \\
\hline 5 & $\begin{array}{l}\text { Machine Learning for Precision } \\
\text { Medicine Forecasts and Challenges } \\
\text { when incorporating Non-Omics and } \\
\text { Omics Data }\end{array}$ & $\begin{array}{l}\text { J Susymary and P } \\
\text { Deepalakshmi }\end{array}$ \\
\hline 6 & $\begin{array}{l}\text { Whale Optimization Algorithm } \\
\text { Fused With SVM To Detect Stress } \\
\text { In EEG Signals }\end{array}$ & $\begin{array}{l}\text { Richa Gupta, } \\
\text { M.Afshar Alam, } \\
\text { Parul Agarwal }\end{array}$ \\
\hline 7 & $\begin{array}{l}\text { Uncertainty Query Sampling } \\
\text { Strategies for Active Learning of } \\
\text { Named Entity Recognition Task }\end{array}$ & $\begin{array}{l}\text { Ankit Agrawal, } \\
\text { Sarsij Tripathi, } \\
\text { Manu Vardhan }\end{array}$ \\
\hline 8 & $\begin{array}{l}\text { Multi-objective Techniques for } \\
\text { Feature Selection and Classification } \\
\text { in Digital Mammography }\end{array}$ & $\begin{array}{l}\text { Shankar Thawkar, } \\
\text { Law Kumar Singh, } \\
\text { Munish Khanna }\end{array}$ \\
\hline 9 & $\begin{array}{l}\text { An Intelligent Unsupervised } \\
\text { Technique for Fraud Detection in } \\
\text { Health Care Systems }\end{array}$ & $\begin{array}{l}\text { Kanksha, Aman } \\
\text { Bhaskar, Sagar } \\
\text { Pande, Rahul Malik } \\
\text { and Aditya } \\
\text { Khamparia }\end{array}$ \\
\hline 10 & $\begin{array}{l}\text { Detection of White Blood Cells } \\
\text { Using Optimized qGWO }\end{array}$ & $\begin{array}{l}\text { Prerna Sharma, } \\
\text { Moolchand } \\
\text { Sharma, Divij } \\
\text { Gupta, Nimisha } \\
\text { Mittal }\end{array}$ \\
\hline 11 & $\begin{array}{l}\text { BioSignal Modelling for prediction } \\
\text { of Cardiac Diseases using Intra } \\
\text { Group Selection Method }\end{array}$ & $\begin{array}{l}\text { Hemant } \\
\text { P.Kasturiwale, } \\
\text { Sujata N Kale }\end{array}$ \\
\hline 12 & $\begin{array}{l}\text { Machine Learning for } \\
\text { Psychological Disorder Prediction } \\
\text { in Indians during COVID-19 } \\
\text { Nationwide Lockdown }\end{array}$ & Akshi Kumar \\
\hline
\end{tabular}

\section{About the Editor(s)}

Deepak Gupta received a B.Tech. degree in 2006 from the Guru Gobind Singh Indraprastha University, India. He also received M.E. degree in 2010 from Delhi Technological University, India and Ph. D. degree in 2017 from Dr. APJ Abdul Kalam Technical University,
India. He has completed his Post-Doctoral research at Inatel, Brazil. With 13 years of rich expertise in teaching and two years in the industry, he focuses on rational and practical learning. He has contributed massive literature in Intelligent Data Analysis, BioMedical Engineering, Artificial Intelligence, and Soft Computing. He has served as Editor-in-Chief, Guest Editor, Associate Editor in SCI and various other reputed journals (IEEE, Elsevier, Springer, \& Wiley). He has actively been an organizing end of various reputed International conferences. He has authored/edited 50 books with National/International level publishers (IEEE, Elsevier, Springer, Wiley, Katson). He has published 167 scientific research publications in reputed International Journals and Conferences including 87 SCI Indexed Journals of IEEE, Elsevier, Springer, Wiley.

Oscar Castillo holds a Doctor in Science degree (Doctor Habilitatus) in Computer Science from the Polish Academy of Sciences (with the Dissertation "Soft Computing and Fractal Theory for Intelligent Manufacturing"). He is a Professor of Computer Science in the Graduate Division, Tijuana Institute of Technology, Tijuana, Mexico. Also, he is serving as Research Director of Computer Science and head of the research group on Hybrid Fuzzy Intelligent Systems. Currently, he is President of HAFSA (Hispanic American Fuzzy Systems Association) and Past President of IFSA (International Fuzzy Systems Association). Prof. Castillo is also Chair of the Mexican Chapter of the Computational Intelligence Society (IEEE). He also belongs to the Technical Committee on Fuzzy Systems of IEEE and the Task Force on "Extensions to Type-1 Fuzzy Systems". He is also a member of NAFIPS, IFSA and IEEE. He belongs to the Mexican Research System (SNI Level 3). His research interests are in Type-2 Fuzzy Logic, Fuzzy Control, Neuro-Fuzzy and Genetic-Fuzzy hybrid approaches. He has published over 300 journal papers, 10 authored books, 40 edited books, 200 papers in conference proceedings, and more than 300 chapters in edited books, in total 865 publications according to Scopus ( $\mathrm{H}$ index $=60$ ), and more than 1000 publications according to Research Gate (H index $=72$ in Google Scholar). He has been Guest Editor of several successful Special Issues in the past, like in the following journals: Applied Soft Computing, Intelligent Systems, Information Sciences, Non-Linear Studies, Fuzzy Sets and Systems, JAMRIS and Engineering Letters. He is currently Associate Editor of the Information Sciences Journal, Applied Soft Computing Journal, Engineering Applications of Artificial Intelligence, Granular Computing Journal and the International Journal on Fuzzy 
Systems. Finally, he has been elected IFSA Fellow in 2015 and MICAI Fellow member in 2017. He has been recognized as a Highly Cited Researcher in 2017 and 2018 by Clarivate Analytics because of having multiple highly cited papers in Web of Science.

Ashish Khanna has 16 years of expertise in Teaching, Entrepreneurship, and Research \& Development He received his Ph.D. degree from National Institute of Technology, Kurukshetra. He has completed his M. Tech. and B. Tech. GGSIPU, Delhi. He has completed his post-doc from the Internet of Things Lab at Inatel, Brazil and University of Valladolid, Spain. He has published around 67 SCI-indexed papers in IEEE Transaction, Springer, Elsevier, Wiley and many more reputed Journals with a cumulative impact factor of above 100 . He has around 125 research articles in top SCI/ Scopus journals, confer- ences and book chapters. He is co-author of around 25 edited and textbooks. His research interest includes Distributed Systems, MANET, FANET, VANET, IoT, Machine learning and many more. He is the originator of Bhavya Publications and Universal Innovator Lab. Universal Innovator is actively involved in research, innovation, conferences, startup funding events and workshops. He has served the research field as a Keynote Speaker/Faculty Resource Person/Session Chair/Reviewer/TPC member/post-doctorate supervision. He is convener and Organizer of ICICC conference series. He is currently working at the Department of Computer Science and Engineering, Maharaja Agrasen Institute of Technology, under GGSIPU, Delhi, India. He is also serving as series editor in Elsevier and De Gruyter publishing houses. 